Effect of i nt ensi ty noi se of semiconduct or I aser s on the di gi tal modul at i on characteristics and the bi $t$ error $r$ ate of opt i cal commini cat i on syst ens

\begin{tabular}{|l|l|}
\hline 著者 & Ahned Must af a, Yamada M nor u \\
\hline $\begin{array}{l}\text { j ournal or } \\
\text { publ i cat i on t i t l e }\end{array}$ & Journal of Appl i ed Physi cs \\
\hline vol une & 104 \\
\hline number & 1 \\
\hline year & 2008 01- 01 \\
\hline URL & ht t p: //hdl . handl e. net /2297/11555 \\
\hline
\end{tabular}




\title{
Effect of intensity noise of semiconductor lasers on the digital modulation characteristics and the bit error rate of optical communication systems
}

\author{
Moustafa Ahmed ${ }^{1, a)}$ and Minoru Yamada ${ }^{2}$ \\ ${ }^{1}$ Department of Physics, Faculty of Science, Minia University, 61519 El-Minia, Egypt \\ ${ }^{2}$ Division of Electrical Engineering and Computer Science, Graduate School of Natural Science \\ and Technology, Kanazawa University, Kakuma-machi, Kanazawa 920-1192, Japan
}

(Received 13 January 2008; accepted 23 April 2008; published online 2 July 2008)

\begin{abstract}
This paper presents theoretical evaluation of the digital modulation performance of semiconductor lasers in digital communication systems with gigabit rates. The study is based on numerical integration of the rate equations augmented by a nonreturn-to-zero (NRZ) bit generator. For solitary semiconductor lasers, the performance is evaluated in terms of the eye diagram, turn-on delay jitter (TOJ), and signal-to-noise ratio. In communication systems, the performance is evaluated by the bit error rate (BER) and power penalty induced by the laser noise. Contributions of both the intrinsic fluctuations and bit pattern to the TOJ and BER are assessed. The results show that when the modulation current is low and the semiconductor laser is biased relatively above threshold, the power penalty decreases although the eye diagram is not well open. When the modulation current is high enough, biasing the semiconductor laser far-above threshold achieves both lower power penalty and higher eye-diagram quality. (c) 2008 American Institute of Physics.
\end{abstract}

[DOI: $10.1063 / 1.2949275$ ]

\section{INTRODUCTION}

Semiconductor lasers are popularly employed as radiation sources in optical communication systems. Laser radiation inherently exhibits intensity fluctuations that affect performance of both the device and the communication system. Inclusion of spontaneous emission into the lasing mode and the processes of electron-hole recombination are intrinsic origins of these fluctuations. ${ }^{1-3}$ When the semiconductor laser is subjected to high-speed pseudorandom digital modulation, the bit pattern adds further fluctuations to the intensity levels in both 1 and 0 bits. In digital communication systems, the bit error rate (BER) represents an important figure of merit of the system performance. ${ }^{4}$ The BER is defined as the probability of incorrect identification of either 1 or 0 levels by the decision circuit in the receiver. Such incorrect identification is caused not only by both circuit and detector shot noises but also by the laser intensity noise. ${ }^{4}$

Another inferior dynamic property of the semiconductor laser is the turn-on delay time, which is defined as the time interval between the onset of the electrical pulse and the converted optical pulse. ${ }^{2,5}$ In digital transmission systems, the turn-on delay is not a fixed quantity but exhibits random fluctuations, giving rise to jitter. This turn-on delay jitter (TOJ) is defined as the standard deviation of the random turn-on delay and works to reduce the bit rate. It then acts as a limiting factor to the system performance. ${ }^{6}$ The TOJ comes from both intrinsic intensity noise and the history of the bits preceding each 1 bit in the pseudorandom bit pattern. Simulation of semiconductor laser fluctuations under digital modulation and evaluation of the system BER are essential

\footnotetext{
a) Author to whom correspondence should be addressed. TEL.: 0020118955943. FAX: 0020-862343011. Electronic mail: moustafaahmed66@gmail.com.
}

to understand behavior of the semiconductor laser and estimate the system performance. Special attention should be paid to modulation with nonreturn-to-zero (NRZ) formats as the RZ format has been intensively employed in evaluating the laser modulation performance. ${ }^{7,8}$ The modulation performance of semiconductor lasers is examined qualitatively by the eye diagram, which is constructed by dividing the output waveform into segments of an equal number of bits and overlaying on each others. Enhanced TOJ is seen in the eye diagram as split of the turn-on edge into distinct paths, ${ }^{9}$ and higher degree of eye opening corresponds to lower BER. Therefore, the eye diagram can be used as an indicator of the system performance.

Agrawal and co-worker ${ }^{10,11}$ examined the contribution of laser noise to the BER of a fiber communication system. However, they employed artificial values of the intensity onoff ratio and signal-to-noise ratio (SNR) assuming similar SNR in both 1 and 0 bits. Moreover, they did not differentiate the individual contributions of the intrinsic fluctuations and bit pattern. ${ }^{10,11}$

Most recently, the authors showed that the bit-pattern induced TOJ is enhanced when the bit slot of a NRZ bit stream is shorter than the setting time of the relaxation oscillations. ${ }^{12}$ When the semiconductor laser is biased near threshold, the TOJ is mainly a bit-pattern effect, whereas the intrinsic noise dominates at high bias levels. ${ }^{12}$ A technique to decide the most open part of the eye diagram was proposed basing on optimizing the laser signal $Q$ factor. ${ }^{12}$ In this paper, we continue our work in Ref. 12 presenting evaluation of the laser modulation performance in digital communication systems. The performance is evaluated in terms of the eye diagram, TOJ, and SNR of the laser waveform as well as the BER and noise-induced power penalty of the system. These characteristics are explored as functions of the modu- 
TABLE I. Definitions of symbols appearing in the above equations and their typical values in the considered InGaAsP laser.

\begin{tabular}{ccc}
\hline \hline Symbol & Definition & Value and unit \\
\hline$\lambda_{0}$ & Emission wavelength & $1.55 \mu \mathrm{m}$ \\
$a$ & Tangential gain coefficient & $7.85 \times 10^{-12} \mathrm{~m}^{3} \mathrm{~s}^{-1}$ \\
$\xi$ & Field confinement factor of electric field to the active & 0.2 \\
$V$ & region & \\
$L$ & Volume of the active region & $60 \mathrm{~m}^{3}$ \\
$n_{r}$ & Length of the active region & $250 \mu \mathrm{m}$ \\
$N g$ & Refractive index of the active region & 3.56 \\
$\tau_{\text {in }}$ & Electron number at transparency & $5.31 \times 10^{7}$ \\
$\left|R_{c v}\right|^{2}$ & Electron intraband relaxation time & $0.13 \mathrm{ps}^{-2}$ \\
$N s$ & Squared absolute value of the dipole moment & $9.53 \times 10^{-57} \mathrm{C}^{2} \mathrm{~m}^{2}$ \\
$B_{\text {eff }}$ & Electron number characterizing nonlinear gain & $4.05 \times 10^{7}$ \\
$G_{\text {th }}$ & Effective rate of radiative and nonradiative & $3.9 \times 10^{-16} \mathrm{~m}^{3} \mathrm{~s}^{-1}$ \\
\hline \hline
\end{tabular}

lation current when the laser is biased above threshold. We assess contributions of both the intrinsic fluctuations and bit pattern to the BER. In the calculations, we consider the parameters of a $1.55 \mu \mathrm{m}$ InGaAsP laser and an avalanche photodiode (APD). We compare the BER versus received power curves at different modulation currents when the intensity noise has Gaussian statistics. These curves are used to investigate the power penalty associated with both intrinsic and total noises of the semiconductor laser. Correlation of these system parameters with the SNR and power on-off ratio within the most open part of the eye diagram is demonstrated. When the modulation current is low and the bias current is relatively high, lower power consumption of the system is achieved although the eye diagram is not well open. When the modulation current is high enough, biasing the laser at high levels achieves lower power penalty and higher eye-diagram quality.

\section{THEORETICAL MODEL}

\section{A. Laser rate equations}

The dynamics of semiconductor lasers under direct digital modulation are simulated by numerical integration of the rate equations of the photon number $S(t)$ and injected electron number $N(t):^{12}$

$$
\begin{aligned}
& \frac{d S}{d t}=\left(G-G_{\mathrm{th}}\right) S+\frac{a \xi}{V} N+F_{S}(t), \\
& \frac{d N}{d t}=\frac{1}{e} I(t)-A S-\frac{N}{\tau_{s}}+F_{N}(t),
\end{aligned}
$$

where $G$ is the optical gain (per second), which is described by the nonlinear form ${ }^{13,14}$

$$
G=A-B S,
$$

where $A$ and $B$ are the linear and nonlinear (suppressed) gain coefficients and are given by

$$
A=\frac{a \xi}{V}\left(N-N_{g}\right),
$$

$$
B=\frac{9}{2} \frac{\pi c}{\varepsilon_{0} n_{r}^{2} \hbar \lambda_{0}}\left(\frac{\xi \tau_{\text {in }}}{V}\right)^{2} a\left|R_{c v}\right|^{2}\left(N-N_{s}\right),
$$

with the term $B S$ measuring the gain suppression which increases the damping rate of the relaxation oscillation. ${ }^{14}$ The last terms $F_{S}(t)$ and $F_{N}(t)$ in Eqs. (1) and (2) are Langevin noise sources and are added to the equations to describe the intrinsic fluctuations in $S(t)$ and $N(t)$ associated with quantum transitions of electrons between the valence and conduction bands. These noise sources have Gaussian statistics with zero means and are $\delta$ correlated. Techniques of simulating these noise sources using computer random-number generations can be found in Ref. 3 .

The direct digital modulation is included in the current term $I(t)$, which represents a stream of coded bits of the modulating electrical signal:

$$
I(t)=I_{b}+I_{m} f_{m}(t) .
$$

$I_{b}$ is the dc-bias current of the laser and $I_{m}$ is the modulation current which decides the modulation depth of the laser. $f_{m}(t)$ is a time varying function with either 0 or 1 level describing the bit format of the modulating current; it commonly describes either a NRZ or a RZ pseudorandom bit generation.

The power $P_{L}(t)$ emitted from the laser front facet is calculated from $S(t)$ via the relationship ${ }^{3}$

$$
P_{L}(t)=\frac{\hbar \pi c^{2}}{n_{r} L \lambda_{0}} \frac{\left(1-R_{f}\right) \ln \left(1 / R_{f} R_{b}\right)}{\left(1-\sqrt{\mathrm{R}_{\mathrm{f}} R_{b}}\right)\left(1-\sqrt{R_{f} / R_{b}}\right)} S(t) .
$$

The signal-to-noise ratio $(\mathrm{SNR}) \mathrm{SNR}_{j}$ at level $1(j=1)$ or level $0(j=0)$ are determined from the corresponding standard deviations of power fluctuation $\sigma_{L(1)}$ or $\sigma_{L(0)}$ and average powers $\bar{P}_{L(1)}$ or $\bar{P}_{L(0)}$, respectively, as

$$
\mathrm{SNR}_{j}=\frac{\bar{P}_{L(j)}}{\sigma_{L(j)}}, \quad j=0 \text { or } 1 .
$$

Definitions of the symbols appearing in the above equations and their typical values in $1.55 \mu \mathrm{m}$ InGaAsP lasers are given in Table I. Other symbols include $e$ as the electron 
charge, $\varepsilon_{0}$ and $c$ as permittivity and speed of light in the free space, respectively, and $\hbar$ as the reduced Planck constant.

\section{B. Bit error rate}

When considering an APD at the receiver end of a communication system with an incident bit rate $B$, the detected signals $\mu_{1}$ and $\mu_{0}$ as the 1 and 0 levels, respectively, represent the detected photocurrents given by ${ }^{10,11}$

$$
\mu_{j}(t)=R_{0}\langle M\rangle P_{j}(t), \quad j=0 \text { or } 1,
$$

where $P_{j}$ is the received power level in either logic levels, $\langle M\rangle$ is the average APD gain, and

$$
R_{0}=e \eta_{d} / h \nu
$$

is the detector responsivity with $\eta_{d}$ being the quantum efficiency. The BER is measured by the decision circuit as the probability of incorrect identification of the 1 and 0 levels of the detected signal, which occurs when the noise level exceeds the threshold decision of either level. Therefore, evaluation of the BER requires counting the various noise mechanisms that contribute to the signal fluctuations, including circuit noise, shot noise, and laser noise. Deriving an expression for the BER requires specifying the probability density function of the signal fluctuations. A common approximation assumes Gaussian probability distributions of these fluctuations in both the 1 and 0 levels. ${ }^{4,15}$ Such an approximation is valid as long as the system does not exhibit strong bitpatterning effects. The Gaussian probabilities $P(1 / 0)$ and $P(0 / 1)$ of incorrect counting of the 1 and 0 bits are characterized by the mean values $\bar{\mu}_{1}$ and $\bar{\mu}_{0}$ and variances $\sigma_{1}^{2}$ and $\sigma_{0}^{2}$, respectively. The variance of the signal fluctuations can be written as a summed value of the variances of the contributing Gaussian noise sources. For a NRZ bit stream, this variance is, ${ }^{4,10}$

$$
\sigma_{j}^{2}=\sigma_{c}^{2}+R_{0}\left\langle M^{2}\right\rangle e B P_{\mathrm{rec}(j)}+R_{0}^{2}\langle M\rangle^{2} \sigma_{L(j)}^{2}, \quad j=0 \text { or } 1 .
$$

The first term represents the circuit noise associated with the thermal and dark-current effects. It is evaluated from the receiver absolute temperature $T$ and dark current $I_{d}$ as ${ }^{4}$

$$
\sigma_{c}^{2}=\left(e\left\langle M^{2}\right\rangle I_{d}+2 \frac{k_{B} T}{R_{L}} F_{n}\right) B,
$$

where $k_{B}$ is the Boltzmann constant, $R_{L}$ is the load resistor, and $F_{n}$ is a factor by which thermal noise is enhanced by resistors in pre- and main amplifiers. The second term in Eq. (11) represents the shot noise, whereas the last term incorporates the laser intensity noise. $P_{\operatorname{rec}(j)}$, where $j=0$ or 1 , is the time average received power defining the average received power $P_{\text {rec }}$ in the receiver as

$$
P_{\text {rec }}=\left(P_{\text {rec }(0)}+P_{\text {rec }(1)}\right) / 2 \text {. }
$$

For NRZ bit codes, these probabilities $P(1 / 0)$ and $P(0 / 1)$ decide the BER as

$$
\mathrm{BER}=\frac{1}{2}[P(0 / 1)+P(1 / 0)] .
$$

The BER is then determined from the system $Q$ factor: ${ }^{16}$

$$
Q=\frac{\bar{\mu}_{1}-\bar{\mu}_{0}}{\sigma_{1}+\sigma_{0}},
$$

as

$$
\mathrm{BER}=\frac{1}{2} \operatorname{erfc}\left(\frac{Q}{\sqrt{2}}\right) \approx \frac{\exp \left\{-Q^{2} / 2\right\}}{Q \sqrt{2} \pi},
$$

where erfc denotes the complementary error function.

\section{PROCEDURES OF NUMERICAL CALCULATIONS}

The rate equations (1) and (2) are solved numerically by means of the fourth-order Runge-Kutta method assuming square pulses of the modulating current in Eq. (6). The counted bit rate is $2.5 \mathrm{Gbits} / \mathrm{s}$ corresponding to bit slot $T_{b}$ $=1 / B=400 \mathrm{ps}$. The time step of integration $\Delta t$ is set as short as $2 \mathrm{ps}$, which corresponds to a cut-off Fourier frequency $(=1 / \Delta t)$ of the laser waveform much higher than the relaxation frequency $f_{r}$.

The laser is modulated with a NRZ pseudorandom bit stream generated by the uniformly distributed randomnumber generations of the computer; random values less than 0.5 are converted to zero and other values are converted to unity. A total number of $2^{12}$ bits are generated and employed in the calculations in order to collect significant statistics of laser intensity. The modulation characteristics are simulated as functions of both $I_{b}$ and $I_{m}$. The calculations are applied to a Fabry-Pérot InGaAsP laser emitting with $\lambda$ $=1.55 \mu \mathrm{m}$ using the parametric values given in Table I. The nonradiative recombination processes are taken into account in the rate equation through the electron lifetime $\tau_{s}$ as ${ }^{12}$

$$
1 / \tau_{s}=B_{\text {eff }} N / V,
$$

where $B_{\text {eff }}$ is the effective rate of radiative and nonradiative recombination processes of electrons.

For evaluation of the BER, we assume the following set of parameters corresponding to a real optic-fiber transmission link as in Ref. 12. A $1.55 \mu \mathrm{m}$ InGaAsP laser diode is assumed to radiate the link with an APD. The APD is assumed with dark current $I_{d}=90 \mathrm{nA}$, average gain $\langle M\rangle=9.1$, quantum efficiency $\eta_{d}=0.68$, and responsivity $R_{0}=0.84$ The second moment $\left\langle M^{2}\right\rangle$ of the APD gain is approximated by $\langle M\rangle^{2} F(\langle M\rangle),{ }^{10,11}$ where $F$ is an excess noise factor given by

$$
F(\langle M\rangle)=k\langle M\rangle+\left(2-\langle M\rangle^{-1}\right)(1-k) .
$$

$k$ is the ionization-coefficient ratio and is set as $k=0.35$. Other parameters of the APD are $F_{n}=2$ and $R_{L}=1 \mathrm{k} \Omega$. The received power $P_{\text {rec }}$ is varied between -40 and $-10 \mathrm{mdB}$ defining $P_{\text {rec }(1)}$ and $P_{\text {rec }(0)}$ as functions of the average power on-off ratio $\bar{P}_{L(10)}=\bar{P}_{L(1)} / \bar{P}_{L(0)}$ of the laser waveform as

$$
\begin{aligned}
& P_{\text {rec }(1)}=2 \bar{P}_{L(10)} P_{\text {red }} /\left(1+\bar{P}_{L(10)}\right), \\
& P_{\text {rec }(0)}=2 P_{\text {rec }} /\left(1+\bar{P}_{L(10)}\right) .
\end{aligned}
$$

Variation in $P_{\text {rec }}$ may be achieved in experiment using an attenuator or a rotating disk with varied transmittance. The standard variations $\sigma_{L(j)}$ in the on and off states as well as 


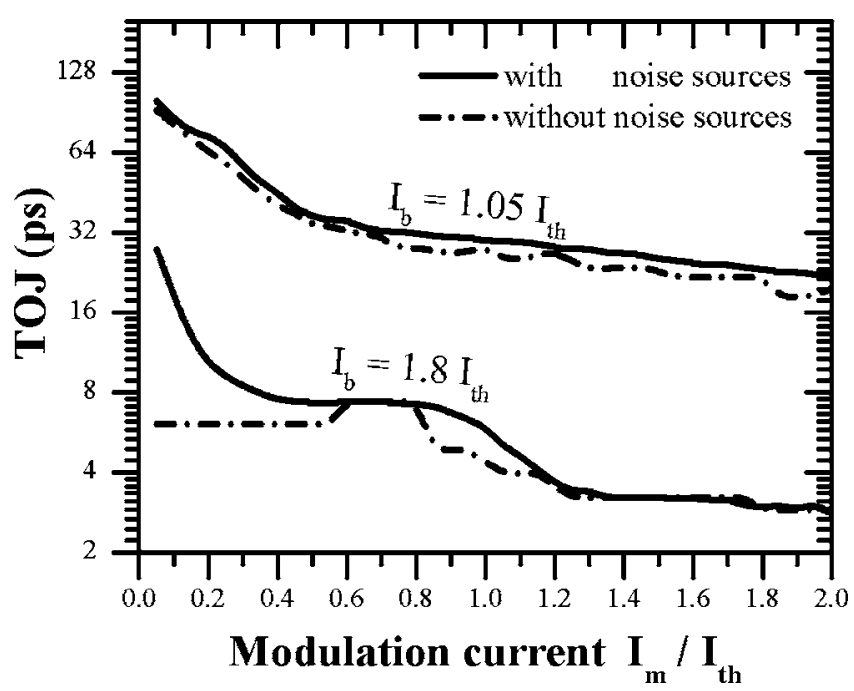

FIG. 1. Variation in TOJ with $I_{m}$ when $I_{b} / I_{\mathrm{th}}=1.05$ and 1.8 . The cases of dropping the noise sources are plotted with dashed lines. TOJ is higher when $I_{b}=1.05 I_{\mathrm{th}}$ than when $I_{b}=1.8 I_{\mathrm{th}}$.

$\bar{P}_{10}$ are determined within the most open sampling period of the eye diagram.

\section{SIMULATION RESULTS AND DISCUSSION}

\section{A. TOJ and eye diagram}

The TOJ is an important indicator of the quality of the modulated laser waveform. The turn-on delay is defined as the time at which the power $P_{L(1)}(t)$ in the 1 bits first surpasses $50 \%$ of the summation of the steady state powers corresponding to $I_{b}$ and $I_{m}$, i.e., $P_{L}(t)$ crosses the middle of the eye diagram. ${ }^{12}$ Figure 1 (a) plots the dependence of the TOJ on $I_{m}$ at two bias levels above threshold, $I_{b}=1.05$ and $3.0 I_{\mathrm{th}}$. The figure shows that the TOJ decreases with the increase in $I_{m}$ with this decrease being bigger at lower values of $I_{m}$. The range of TOJ when $I_{b}=1.05 I_{\mathrm{th}}(22-100 \mathrm{ps})$ is much higher than that $(3-27 \mathrm{ps})$ when $I_{b}=1.05 I_{\mathrm{th}}$. This is because the relaxation oscillations in the 0 level are much enhanced near the threshold level, boosting the random effect of the bit pattern on the TOJ. ${ }^{9,12}$ However, these oscillations are much damped with the increase in $I_{b}$, which moderates the random effect of the bit pattern on TOJ in both 0 and 1 levels. To examine the influence of intrinsic noise on the explored results, we plot in the figure with dashed lines the corresponding variations in the TOJ when the intrinsic

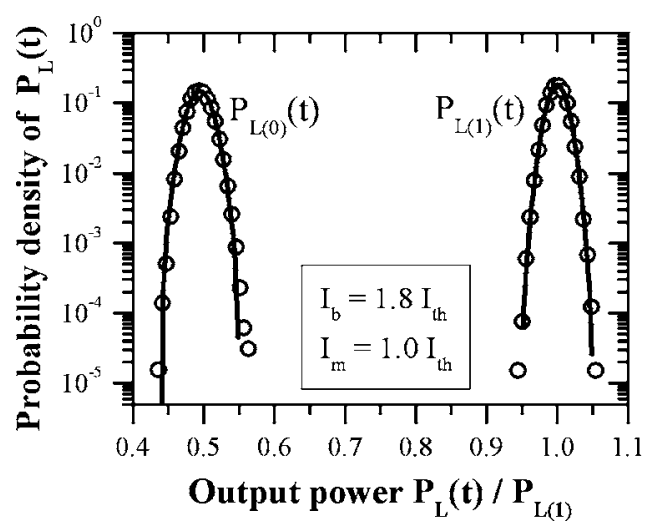

FIG. 3. Probability density of $P_{L(0)}(t)$ and $P_{L(1)}(t)$ when $I_{b} / I_{\mathrm{th}}=1.8$ and $I_{m} / I_{\text {th }}=1$ within the most open portion of the eye diagram. The data are well fitted by the Gaussian distribution represented by the solid lines.

noise is ignored. This corresponds to the case of dropping the noise sources in rate equations (1) and (2). The figure shows that ignoring the intrinsic noise slightly decreases TOJ over the relevant range of $I_{m}$ when $I_{b}=1.05 I_{\mathrm{th}}$. On the other hand, the corresponding drop in TOJ in the case of $I_{b}=1.8 I_{\mathrm{th}}$ is significant in the low range of $I_{m} \leqslant 0.5 I_{\text {th }}$ but is almost negligible in the high range of $I_{m}$. These results reflect the major contribution of the intrinsic noise to the TOJ at low modulation currents when the laser is biased far-above threshold.

The eye diagram can be used to examine qualitatively the random phenomenon of the turn-on delay at different levels of biasing and modulation. Figures 2(a) $-2(\mathrm{~d})$ plot the 3 bit long eye diagrams of $P_{L}(t)$ when $I_{b}=1.05 I_{\text {th }}$ and Figs. $2(\mathrm{e})-2(\mathrm{~h})$ plot the eye diagrams when $I_{b}=1.8 I_{\mathrm{th}}$, when $I_{m} / I_{\text {th }}=0.5,1.0,1.5$, and 2 , respectively. Figures $2(\mathrm{a})-2(\mathrm{~d})$ demonstrate the enhanced random effect of the turn-on delay characterizing the case of biasing the laser near-above threshold. The laser takes various paths to reach the 1 level, showing also budges on the top of the eyes. This phenomenon is a pseudorandom bit-pattern effect because the setting time of the relaxation oscillations is $1.2 \mathrm{~ns}$ at $I_{b}=1.05 I_{\mathrm{th}}$, which is almost three times longer than the bit slot $T_{b}{ }^{12}$ The increase in $I_{m}$ causes an increase in the intensity of the 1 level, which results in more opening (i.e., improvement) of the eye diagram. In the case of $I_{b}=1.8 I_{\mathrm{th}}$, the setting time of the relaxation oscillations is $0.272 \mathrm{~ns}$, which is shorter than $T_{b}$; therefore, the random effect of the turn-on delay is much suppressed as shown in Figs. 2(e)-2(h). The figures show also that the power level in the 1 state increases and the

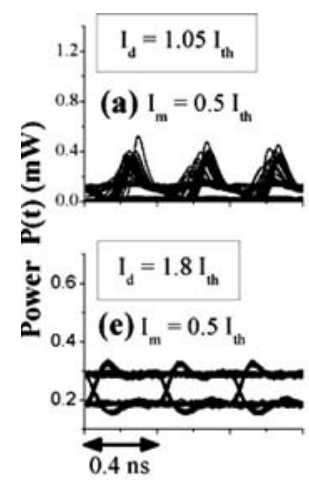

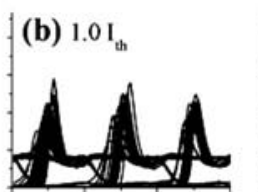
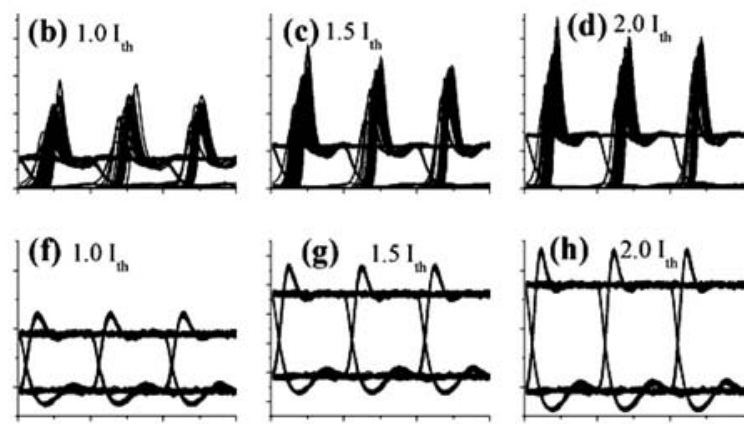

FIG. 2. Simulated eye diagrams when $[(\mathrm{a})-(\mathrm{d})] I_{b} / I_{\text {th }}$ $=1.05$ and $[(\mathrm{e})-(\mathrm{h})] I_{b} / I_{\mathrm{th}}=1.8$ for $I_{m} / I_{\mathrm{th}}=0.5,1.0,1.5$, and 2.0, respectively. The TOJ is enhanced when $I_{b} / I_{\text {th }}=1.05$ where the laser power follows different paths to reach the 1 level. The eye diagrams become more open with the increase in $I_{m}$. 


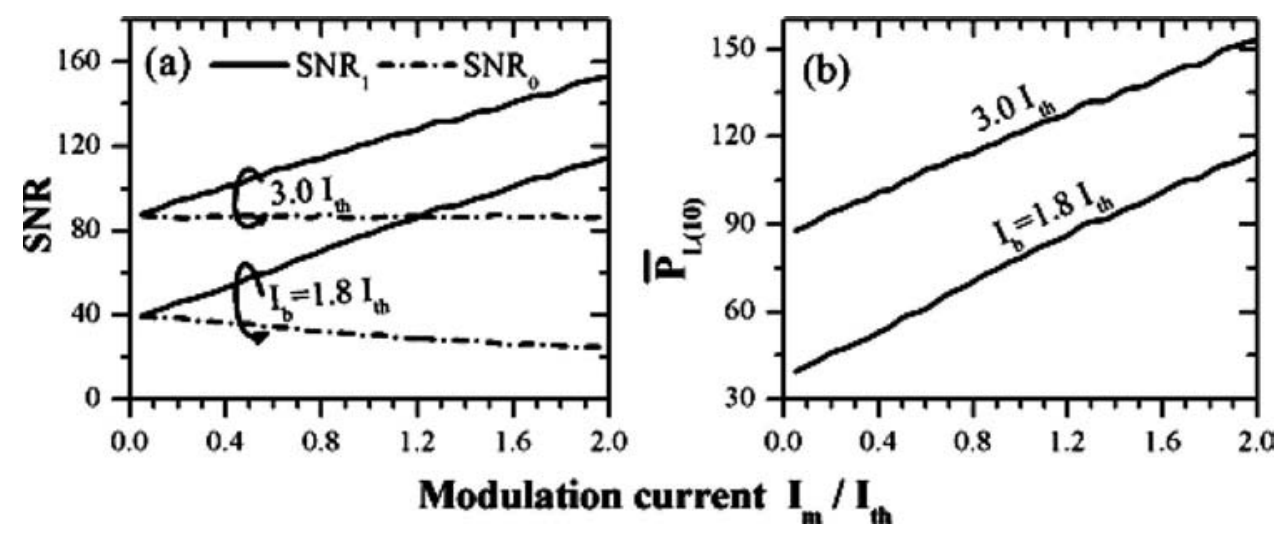

FIG. 4. Influence of $I_{m}$ on (a) $\mathrm{SNR}_{0}$ and $\mathrm{SNR}_{1}$ and (b) $\bar{P}_{L(10)}$ when $I_{b}$ $=1.8 I_{\text {th }}$ and $3.0 I_{\text {th. }}$. Both $\mathrm{SNR}_{1}$ and $P_{L(10)}$ improve with the increase in $I_{m}$ while $\mathrm{SNR}_{0}$ decreases slightly. associated power fluctuations is suppressed with the increase in $I_{m}$, which corresponds to more opening (improvement) of the eye diagram.

\section{SIGNAL-TO-NOISE RATIO AND POWER ON-OFF RATIO}

As seen in Eqs. (8), (11), (19), and (20), the parameters of the modulated laser waveform that determines the BER of a communication system are $\mathrm{SNR}_{1}, \mathrm{SNR}_{0}$ and $\bar{P}_{L(10)}$. These statistical parameters are usually determined within the mostopen part of the eye diagram. This part is determined following the technique proposed by the authors in Ref. 12. The laser signal is sampled over different periods within the bit slot $T_{b}$ and at different decision times. The most open part of the eye diagram then corresponds to the sampling and decision times that yield the highest value of the laser signal $Q$ factor defined as

$$
Q_{\text {signal }}=\frac{\bar{P}_{L(1)}-\bar{P}_{L(0)}}{\sigma_{L(0)}+\sigma_{L(1)}} .
$$

We pick up two bias currents, $I_{b} / I_{\mathrm{th}}=1.8$ (relatively above threshold) and 3.0 (far-above threshold). The optimized sampling and decision times are 0.04 and $0.36 \mathrm{ns,}$ respectively, over which the laser power fluctuations have Gaussian probability distributions. An example confirming this Gaussian statistics is illustrated in Fig. 3, which plots the probability density function of the fluctuating powers $P_{L(0)}(t)$ and $P_{L(1)}(t)$ in both signal levels when $I_{b}=1.8 I_{\text {th }}$ and $I_{m}=I_{\mathrm{th}}$. The figure shows good fit of the sampled power fluctuations with Gaussian distributions, which are represented by solid lines. Since the statistics of both thermal and shot noises are described by Gaussian probability distributions, ${ }^{4}$ this Gaussian fit validates application of the present model of BER calculation at the chosen bias currents.

Figure 4(a) plots variations in $\mathrm{SNR}_{1}$ and $\mathrm{SNR}_{0}$ with modulation current $I_{m}$, while Fig. 4(b) plots the corresponding variation in $\bar{P}_{L(10)}$. Figure $4(\mathrm{a})$ shows that $\mathrm{SNR}_{1}$ increases almost linearly with $I_{m}$ while $\mathrm{SNR}_{0}$ decreases much more slightly. Moreover, the range of $\mathrm{SNR}_{1}$ is considerably higher than that of $\mathrm{SNR}_{0}$ especially at higher modulation currents $I_{m}$. The artificial setting of $\mathrm{SNR}_{0}=\mathrm{SNR}_{1}$ and the consequent analysis of BER reported by Shen and Agrawal $^{11}$ may then fit the low range of $I_{m}$ in the present analysis. The obtained results indicate that the laser noise is almost determined by the power fluctuations in the 1 level. Figure 4(b) shows that $\bar{P}_{L(10)}$ increases also linearly with the increase in $I_{m}$. Figures 4(a) and 4(b) show also that $\mathrm{SNR}_{0}, \mathrm{SNR}_{1}$, and $\bar{P}_{L(10)}$ are higher for $I_{b}=3.0 I_{\mathrm{th}}$ than for $I_{b}=1.8 I_{\mathrm{th}}$. This is because the increase in $I_{b}$ is associated with improvement in the eyediagram quality in terms in reduction in intensity noise and increase in the 1 level, as discussed in the previous subsection.

\section{A. BER and power penalty due to laser noise}

In order to gain insight on how to control the laser diode and improve its performance in digital communication systems, it is essential to examine influences of both the bias and modulation currents on the system BER. Figures 5(a) and 5(b) plot the dependence of the characteristic curves of BER versus $P_{\text {rec }}$ at different modulation currents $I_{m}$ when
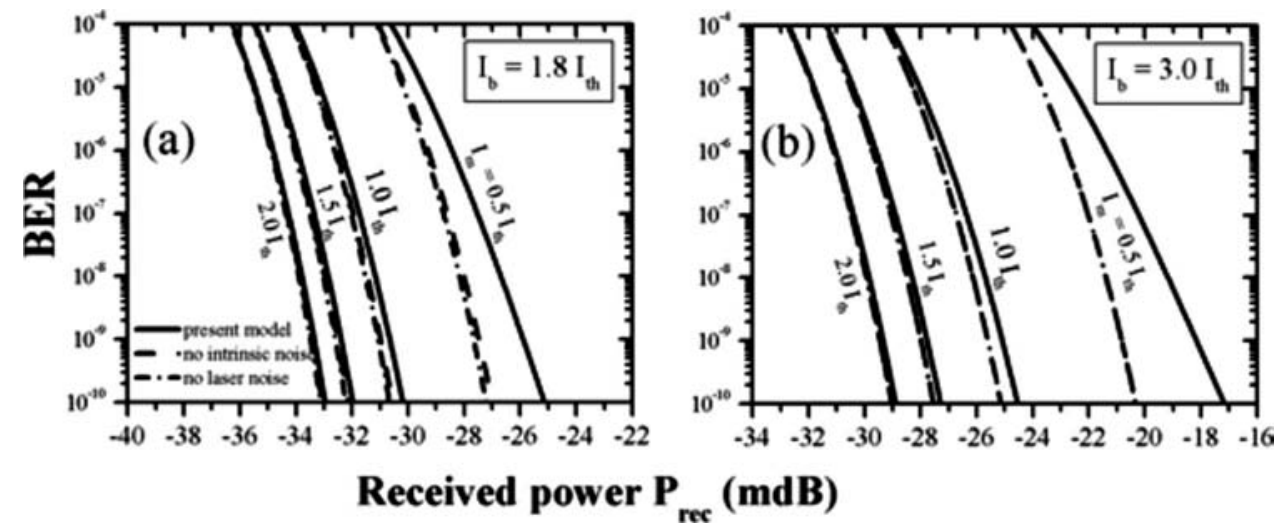

FIG. 5. Characteristic curves of BER vs $P_{\text {rec }}$ of the considered communication system as functions of $I_{m}$ when (a) $I_{b}=1.8 I_{\mathrm{th}}$ and (b) $I_{b}=3.0 I_{\mathrm{th}}$. The BER drops to lower orders of magnitude with the increase in $P_{\text {rec }}$.

\section{Received power $\mathbf{P}_{\text {rec }}(\mathbf{m d B})$}




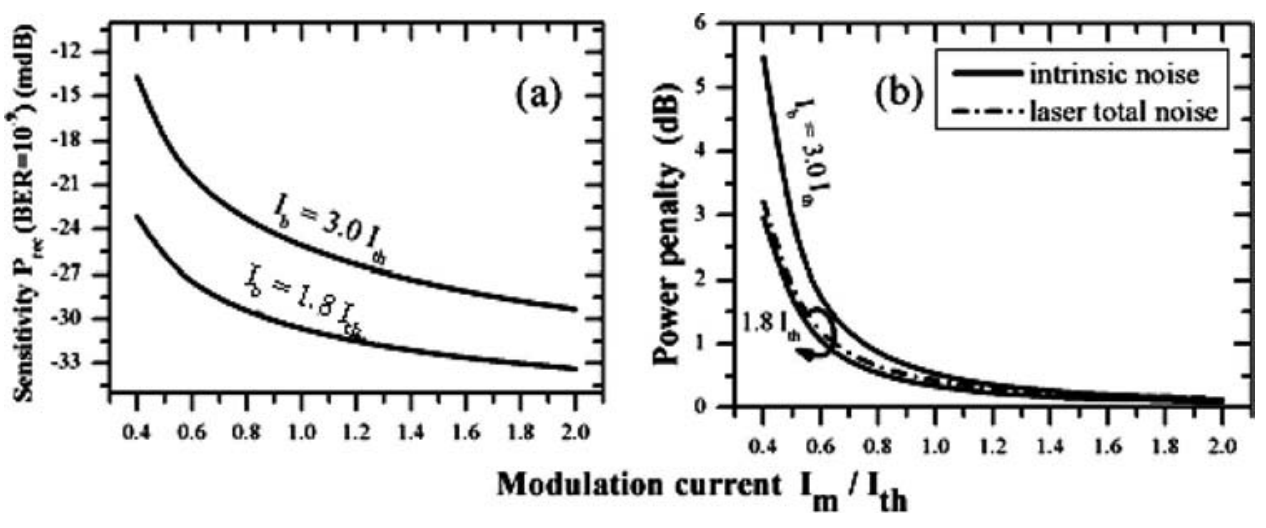

FIG. 6. Influence of $I_{m}$ on (a) receiver sensitivity and (b) induced power penalty by intrinsic and total noises of the laser when $I_{b}=1.8$ and $I_{b}=3.0 I_{\mathrm{th}}$. Both sensitivity and power penalty are higher when $I_{b}=1.8 I_{\text {th }}$; however, they are improved more with the increase in $I_{m}$ when $I_{b}=3.0 I_{\mathrm{th}}$.
$I_{b}=1.8 I_{\text {th }}$ and $3 I_{\text {th }}$, respectively. Either figure shows the typical character of dropping the BER to lower orders of magnitude with the increase in $P_{\text {rec }}{ }^{4,10,11}$ This drop of the BER becomes stronger at higher magnitudes of $I_{m}$.

The laser power fluctuations that contribute to the BER originate from the coupled effect of the intrinsic intensity fluctuations and the pseudorandom bit pattern. In Fig. 5, we assess these contributions by plotting the corresponding BER characteristics when (a) the intrinsic intensity noise of the laser is ignored (dashed lines), i.e., the noise sources $F_{S}(t)$ and $F_{N}(t)$ are dropped in rate equations (1) and (2), and (b) the laser total noise is ignored (dash-dot lines), i.e., setting $\sigma_{L}=0$ in Eq. (11). Figure 5(a) shows that in the low range of $I_{m}$, ignoring the intrinsic noise causes significant drop in the BER. This effect diminishes with the increase in $I_{m}$. When $I_{b}=1.8 I_{\text {th }}$, Fig. 5(a) shows that ignoring the laser total noise causes more drop in the BER than the case of ignoring the intrinsic noise only when $I_{m}<I_{\text {th }}$. At higher modulation currents, both effects yield the same BER characteristics. That is, the pseudorandom bit pattern affects the BER performance at relatively low values of $I_{m}$. On the other hand, when $I_{b}=3.0 I_{\text {th }}$, Fig. 5(b) shows that ignoring the laser total noise has the same effect on the BER over the relevant range of $I_{m}$. This indicates that the pseudorandom bit pattern has no influence on the BER performance when the laser is biased far above the threshold level.

It is conventional to measure the BER performance of the system in terms of the receiver sensitivity, defined as the amount of received power $P_{\text {rec }}$ required to achieve a specific BER level. ${ }^{4}$ The variations in the sensitivity at a BER floor of $10^{-9}$ with $I_{m}$ corresponding to Fig. 5 are plotted in Fig. 6(a). The figure shows that the sensitivity is higher when $I_{b}$ $=3.0 I_{\text {th }}$ than when $I_{b}=1.8 I_{\text {th }}$ over the relevant range of $I_{m}$. However, the associated improvement in sensitivity with the increase in $I_{m}$ is better at the higher bias level; sensitivity improves from -13.7 to $-29.3 \mathrm{mdB}$ when $I_{b}=3.0 I_{\mathrm{th}}$ but from -23.1 to $-33.4 \mathrm{mdB}$ when $I_{b}=1.8 I_{\mathrm{th}}$.

The sensitivity data in Fig. 6(a) are used to estimate the system power penalty at $\mathrm{BER}=10^{-9}$ induced by both the intrinsic and total intensity noises of the laser. These power penalties are defined as

power penalty due to intrinsic noise $(\mathrm{dB})$

$$
=10 \log 10\left(\frac{P_{\mathrm{rec}}}{P_{\mathrm{rec}}\left(F_{S}(t)=F N(t)=0\right)}\right),
$$

power penalty due to total noise $(\mathrm{dB})$

$$
=10 \log 10\left(\frac{P_{\mathrm{rec}}}{P_{\mathrm{rec}}\left(\sigma_{L(j)}=0\right)}\right),
$$

respectively, and are plotted in Fig. 6(b). The figure shows that both power penalties are the same when $I_{b}=3.0 I_{\text {th }}$ because the intrinsic noise is the main contributor to the laser noise as discussed above. When $I_{b}=1.8 I_{\text {th }}$, however, the power penalty induced by the laser total noise is a little higher than that due to the intrinsic noise as a manifestation of the pseudorandom bit pattern. Although the induced power penalty is higher when $I_{b}=3.0 I_{\text {th }}$ than when $I_{b}$ $=1.8 I_{\text {th }}$, it improves better with the increase in $I_{m}$ at the higher bias level. The above results indicate that the noise (BER) performance of the laser in communications systems improves with the increase in $I_{m}$. When $I_{m}$ is relatively low, biasing the laser relatively above threshold is beneficial to achieve power consumption of the system although the corresponding eye diagrams are not well open. However, at higher modulation currents, it is preferred to operate the laser far-above threshold to achieve both lower power penalty and higher eye-diagram quality.

\section{CONCLUSIONS}

Theoretical evaluation of semiconductor laser performance in communication systems under NRZ-digital modulation with a gigabit rate has been presented. The intrinsic performance of the laser was evaluated in terms of the eye diagram, TOJ, SNR, and power on-off ratio. The performance in the communication system was evaluated in terms of the BER and power penalty raised by the laser noise. The obtained results can be concluded as follows:

(1) Increasing the modulation current causes (a) decrease in the TOJ, (b) improvement in the eye quality in terms of increase in both the SNR and power on-off ratio, and (c) improvement in the system BER.

(2) When the laser is biased near-above threshold, the TOJ is much increased due to the significant random effect of the bit pattern. This appears in the eye diagram as various paths taken by the laser power to reach the 1 level.

(3) When the bias level increases such that the setting time of the relaxation oscillations is shorter than the bit slot, the TOJ decreases and the eye diagram is well open.

(4) Low modulation current with relatively high bias current 
corresponds to low power penalty (system power consumption) although the eye diagram is not well open.

(5) When the modulation current is high enough, operating the laser far-above threshold achieves both lower power penalty and higher eye-diagram quality.

\section{ACKNOWLEDGMENTS}

The first author wishes to thank Doctor Richard Schatz at KTH, Sweden, and Doctor Johan Gustavsson at Chalmers University of Technology, Sweden, for helpful discussions.

${ }^{1}$ D. Marcuse, IEEE J. Quantum Electron. QE-20, 1148 (1984).

${ }^{2}$ G. P. Agrawal and N. K. Dutta, Semiconductor Lasers (Van Nostrand Reinhold, New York, 1993).

${ }^{3}$ M. Ahmed, M. Yamada, and M. Saito, IEEE J. Quantum Electron. 37, 1600 (2001).
${ }^{4}$ G. P. Agrawal, Fiber-Optic Communication Systems (Wiley, New York, 2002).

${ }^{5}$ K. Petermann, Laser Diode Modulation and Noise (Kluwer Academic, Dordrecht, 1988).

${ }^{6}$ G. P. Agrawal and T. M. Shen, Electron. Lett. 22, 450 (1986).

${ }^{7}$ C. Mirasso, P. Colet, and M. San Miguel, Opt. Lett. 16, 1753 (1991).

${ }^{8}$ S. R. Mirasso, P. Colet, and M. San Miguel, IEEE J. Quantum Electron. 29, 23 (1993).

${ }^{9}$ J. S. Gustavsson, A. Haglund, J. Bengtsson, and A. Lasrsson, IEEE J. Quantum Electron. 38, 1089 (2002).

${ }^{10}$ G. P. Agrawal and T. M. Shen, J. Lightwave Technol. LT-4, 58 (1986).

${ }^{11}$ T. M. Shen and G. P. Agrawal, J. Lightwave Technol. LT-5, 653 (1987).

${ }^{12}$ M. Ahmed, M. Yamada, and S. W. Z. Ahmoud, J. Appl. Phys. 101, 033119 (2007).

${ }^{13}$ M. Yamada and Y. Suematsu, J. Appl. Phys. 52, 2653 (1981).

${ }^{14}$ S. Abdulrhmann, M. Ahmed, and M. Yamada, Opt. Rev. 9, 260 (2002).

${ }^{15}$ R. G. Smith and S. D. Personik, Semiconductor Devices for Optical Communication Systems, edited by H. Kressel (Springer-Verlag, Berlin, 1982).

${ }^{16}$ N. S. Bergano, F. W. Kerfoot, and C. R. Davidson, IEEE Photonics Technol. Lett. 5, 304 (1993). 